\title{
NONLINEAR NETWORKS. IIa
}

\section{R. J. DUFFIN}

A network is a collection of conducting wires and batteries arbitrarily interconnected. Kirchhoff $[1,6]^{1}$ gave a topological-type proof that the currents in the wires are uniquely determined for wires obeying Ohm's law. (Ohm's law is a linear law stating that current and potential drop are proportional.) If the wires obey a nonlinear law, more than one distribution of current is in general possible. For some engineering application a multiplicity of states is desirable as, for example, in counting circuits and oscillators. For other applications it is essential that only one state be possible. It is seldom intuitively evident, however, whether or not a given nonlinear network will have multiple states. Hence, it appears that a qualitative mathematical treatment of nonlinear networks should be of some practical importance [5].

A large class of conductors used in engineering are such that the current through the conductor and the potential drop across the conductor are nondecreasing functions of one another. Such conductors we shall term quasi-linear. Examples are: selenium, copper oxide, silicon carbide (thyrite), and thermionic rectifiers [9]. The main result of this note is the proof that $a$ network of quasi-linear conductors has a stable state of currents, and this state is unique.

A stable state of currents in a network must satisfy Kirchhoff's laws, which simply are statements of the conservation of electricity and the single valuedness of the potential function. Maxwell [8] discovered two concise ways of expressing these laws: the junction equations and the mesh equations. More or less as a digression we shall show that the mesh equations may be put in the same functional form as the junction equations if and only if the network is planar.

The formulation of mechanical analogs to electric networks has received considerable attention in the literature because of the transfer of techniques suggested by the analogy. We discuss here a different type of analog which we call an elastic network. An elastic network is a collection of springs connected to each other at junction points. Forces are applied to the junction points to hold the network in a stretched condition. A tennis net is an example. Electric networks are analogous to one-dimensional elastic networks. Planar or spatial

Presented to the Society, September 15, 1945 ; received by the editors September 8 , 1945, and, in revised form, March 18, 1947.

1 Numbers in brackets refer to the references cited at the end of the paper. 
elastic networks are more general than electric networks and may give rise to nonlinear problems even for springs obeying Hooke's law.

1. Maxwell's junction equations. In order to simplify notation and to avoid ambiguity the following trivial restrictions shall be imposed on the configuration of the networks considered. A proper network is a set of $n+1$ junction points $(0,1, \cdots, n), n \geqq 1$, connected by conducting wires such that each wire connects exactly two distinct junction points and no more than one wire directly connects the same two junction points.

The conductivity function $g(x)$ of a wire is the experimentally determined relation between the current $w$ through the wire and the potential drop $x$ across it, $w=g(x)$. If a wire obeys Ohm's law, $g(x)=k x$ where $k$ is a positive constant, the conductivity.

The conductivity function of the wire connecting junction points $i$ and $j$ will be designated as $g_{i j}(x)$ and is such that if $w_{i j}$ is the current flowing from $i$ to $j$ and $v_{i}$ and $v_{j}$ are the potentials of these junctions then

$$
w_{i j}=g_{i j}\left(v_{i}-v_{j}\right) .
$$

By the conservation of electricity, $w_{i j}=-w_{j i}$; so $g_{i j}(x)=-g_{j i}(-x)$. If there is no wire directly connecting $i$ and $j$ then $g_{i j}(x) \equiv 0$.

Suppose currents $u_{i}$ flow into the junction points from an external source. If a stable state of such a network exists, no electricity may pile up at a junction point so

$$
\begin{aligned}
& u_{0}=0+g_{01}\left(v_{0}-v_{1}\right)+g_{02}\left(v_{0}-v_{2}\right)+\cdots+g_{0 n}\left(v_{0}-v_{n}\right), \\
& u_{1}=g_{10}\left(v_{1}-v_{0}\right)+0 \ldots+g_{12}\left(v_{1}-v_{2}\right)+\cdots+g_{1 n}\left(v_{1}-v_{n}\right), \\
& \ldots \ldots \ldots \ldots \ldots \ldots \ldots \ldots \ldots \ldots \ldots \ldots \ldots \ldots \ldots \ldots \ldots
\end{aligned}
$$

These are Maxwell's junction equations. Since $g_{i j}(x)+g_{j i}(-x) \equiv 0$ it follows that $\sum_{i=0}^{n} u_{i} \equiv 0$, and so at least one of the equations is dedependent. Moreover, because the potentials $v_{i}$ enter only as paired differences, one of them may be given an arbitrary value.

If equations (2) are solvable for the potentials, then relations (1) determine the distribution of current in the wires when the currents entering the junction are given.

It should be noted that the definition given of the conductivity function is broad enough to allow batteries to be included in the network. Thus, if for one value of $x$ the relation $g_{i j}(x)=0$ holds, then $-x$ may be regarded as the value of the potential jump of a battery inserted in the wire connecting points $i$ and $j$. Thus, for an isolated 
network excited by batteries equations (2) become

$$
0=\sum_{j=0}^{n} g_{i j}\left(v_{i}-v_{j}\right), \quad i=0,1, \cdots, n .
$$

In a non-proper network two or more wires may directly connect the same junction points. This case may be reduced to a proper network since the conductivity function for wires connected in parallel is the sum of the separate functions. The class of functions to be considered in what follows has the property that the sum of two functions also belongs to the class; hence, there is no essential restriction in considering only proper networks.

2. Existence and uniqueness conditions. The junction equations will now be considered abstractly. However, all variables and constants shall be assumed real.

THEOREM 1. Equations (3) have at least one solution in the variables $v_{i}$ if:

(a) $g_{i j}(x) \equiv-g_{j i}(-x)$.

(b) The functions $g_{i j}(x)$ are continuous for all $x$.

(c) For each pair $(i, j)$ either $g_{i j}(x) \equiv 0$ or $\int_{0}^{x} g_{i j}(t) d t \rightarrow+\infty$ as $x \rightarrow \pm \infty$.

Proof. We shall say that a set of functions $\left\{g_{i j}(x)\right\}$, $i, j=0,1, \cdots, n$, satisfies the chain condition if for each integer $i$ there exists an ordered sequence of integers $i, a, b, c, \cdots, e, f$ (dependent on $i$ ) such that no function of the sequence $g_{i a}, g_{a b}, g_{b c}$, ..., $g_{e f}, g_{f 0}$ vanishes indentically. First, suppose that the chain condition is satisfied and define $G_{i j}(x)=\int_{0}^{x} g_{i j}(t) d t$. Then by conditions (a) and (c) it is clear that there is an unbounded increasing function $h(x)$ such that either $G_{i j}(x) \equiv 0$ or $G_{i j}(x) \geqq h(|x|)$. It may be assumed, moreover, that $h(x)$ is independent of $i$ and $j$ and that $h(0)$ is negative.

Let $v_{0}=0$ and define $\psi\left(v_{1}, v_{2}, \cdots, v_{n}\right)=\sum_{i=0}^{n} \sum_{j=0}^{n} G_{i j}\left(v_{i}-v_{j}\right)$. Consider the value of $\psi$ on an $n$-dimensional cube whose corners are at the points $( \pm l, \pm l, \cdots, \pm l), l>0$. For some value of $i$ it follows that $v_{i}= \pm l$. Hence, $\left(v_{i}-v_{a}\right)+\left(v_{a}-v_{b}\right)+\cdots+\left(v_{e}-v_{f}\right)+\left(v_{f}-v_{0}\right)$ $=\left(v_{i}-v_{0}\right)= \pm l$. All integers of the chain sequence $i, a, b, \cdots, f, 0$ may be assumed distinct; so there are at most $n$ adjacent pairs. Thus, for some adjacent pair, say $(d, e)$, it follows that $\left|v_{d}-v_{e}\right| \geqq l / n$ and $G_{d e}\left(v_{d}-v_{e}\right) \geqq h(l / n)$. An estimate of $\psi$ may be made from this single term, $\psi \geqq h(l / n)+(n+1)^{2} h(0)$. For $l$ sufficiently large this shows that $\psi$ is everywhere greater on the surface of the cube than it is the center. In the interior, $\psi$ must have a minimum point and at this point 


$$
0=2^{-1} \partial \psi / \partial v_{k}=\sum_{j=0}^{n} g_{k j}\left(v_{k}-v_{j}\right), \quad k=1,2, \cdots, n .
$$

These are precisely equations (3) except for the equation $k=0$, which is dependent on the others.

If the chain condition is not satisfied, equations (3) are split into two sets. The first set includes the equation 0 and all equations $i$ such that a nonvanishing chain $g_{i a}, g_{a b}, \cdots, g_{e f}, g_{f 0}$ does exist. If $g_{i x}$ appears in the first set as a nonvanishing function so also does $g_{x i}$, because the chain $g_{x i}, g_{i a}, g_{a b}, \cdots, g_{e f}, g_{f 0}$ is nonvanishing. This shows that the equations and variables appearing in the first set have the same labels. The second set, being what is left over, also has the same labels on the equations and the variables appearing. Clearly both sets have the same form as equations (3) but are of lower dimension than $n$. This splitting process is repeated on the second set, and so on. Finally, there results a division into a number of mutually exclusive sets, and each set either satisfies the chain condition or vanishes identically. This completes the proof.

In the network language the chain condition means that there is a chain of wires connecting every pair of junction points. In other words, the network is one piece.

THEOREM 2. Equations (2) have a solution in the variables $v_{i}$ for any choice of the constants $u_{i}$ such that $\sum_{i=0}^{n} u_{i}=0$ if:

(a) $g_{i j}(x)=-g_{j i}(-x)$.

(b) The function $g_{i j}(x)$ are continuous for all $x$.

(c) For each pair $(i, j)$ either $g_{i j}(x) \equiv 0$ or $g_{i j}(x) \rightarrow+\infty(-\infty)$ as $x \rightarrow+\infty(-\infty)$.

(d) The chain condition is satisfied.

Proof. Define $\psi_{1}=\sum_{i=0}^{n} \sum_{j=0}^{n} G_{i j}\left(v_{i}-v_{j}\right)-2 \sum_{i=0}^{n} u_{i} v_{i}\left(v_{0}=0\right)$. Either $G_{i j}(x) \equiv 0$ or $G_{i j}(x) \geqq h(|x|)$, where now $h(x)$ is an increasing function such that $h(x) / x \rightarrow+\infty$ as $x \rightarrow+\infty$. Proceeding as in the proof of Theorem 1 gives the inequality

$$
\psi_{1} \geqq h(l / n)+(n+1) h(0)-l \sum_{i=1}^{n}\left|u_{i}\right| \rightarrow+\infty \quad \text { as } l \rightarrow+\infty .
$$

This shows that $\psi_{1}$ has a minimum at a point inside a large cube; hence at this point

$$
0=2^{-1} \partial \psi_{1} / \partial v_{k}=\sum_{j=0}^{n} g_{k j}\left(v_{k}-v_{j}\right)-u_{k}, \quad k=1,2, \cdots, n .
$$

This completes the proof. 
THEOREM 3. Equations (2) may not have more than one solution in the variables $v_{i}, i=1,2, \cdots, n$, with $v_{0}=0$ if:

(a) $g_{i j}(x) \equiv-g_{j i}(-x)$.

(b) For each pair $(i, j)$ either $g_{i j}(x) \equiv 0$ or $g_{i j}(x)$ is an increasing function for all $x$.

(c) The chain condition is satisfied.

PROOF. Let $u_{i}=\sum_{j=0}^{n} g_{i j}\left(v_{i}-v_{j}\right)$ and $u_{i}^{\prime}=\sum_{j=0}^{n} g_{i j}\left(v_{i}^{\prime}-v_{j}^{\prime}\right)$ then

$$
\begin{aligned}
& \sum_{i=0}^{n}\left(u_{i}-u_{i}^{\prime}\right)\left(v_{i}-v_{i}^{\prime}\right) \\
& \quad=\sum_{i=0}^{n} \sum_{j=0}^{n}\left\{g_{i j}\left(v_{i}-v_{j}\right)-g_{i j}\left(v_{i}^{\prime}-v_{j}^{\prime}\right)\right\}\left(v_{i}-v_{i}^{\prime}\right) \\
& \quad=\sum_{i=0}^{n} \sum_{j=0}^{n}\left\{g_{j i}\left(v_{i}-v_{i}\right)-g_{j i}\left(v_{j}^{\prime}-v_{i}^{\prime}\right)\right\}\left(v_{j}-v_{j}^{\prime}\right) \\
& \quad=\sum_{i=0}^{n} \sum_{j=0}^{n}\left\{g_{i j}\left(v_{i}-v_{j}\right)-g_{i j}\left(v_{i}^{\prime}-v_{j}^{\prime}\right)\right\}\left(v_{j}^{\prime}-v_{j}\right) \\
& \quad=2^{-1} \sum_{i=0}^{n} \sum_{j=0}^{n}\left\{g_{i j}\left(v_{i}-v_{j}\right)-g_{i j}\left(v_{i}^{\prime}-v_{j}^{\prime}\right)\right\}\left\{\left(v_{i}-v_{j}\right)-\left(v_{i}^{\prime}-v_{j}^{\prime}\right)\right\} .
\end{aligned}
$$

If $u_{i}=u_{i}^{\prime}$, the left side vanishes. Each term of the last series on the right side is non-negative, because $g_{i j}$ is a nondecreasing function; hence,

$$
\left\{g_{i j}\left(v_{i}-v_{j}\right)-g_{i j}\left(v_{i}^{\prime}-v_{j}^{\prime}\right)\right\}\left\{\left(v_{i}-v_{j}\right)-\left(v_{i}^{\prime}-v_{j}^{\prime}\right)\right\}=0 .
$$

Under the assumption that $g_{i j}$ is an increasing function, these factors must vanish together, so $\left\{\left(v_{i}-v_{j}\right)-\left(v_{i}^{\prime}-v_{j}^{\prime}\right)\right\}=0$. For a chain sequence,

$$
\begin{aligned}
\left\{\left(v_{i}-v_{a}\right)-\left(v_{i}^{\prime}-v_{a}^{\prime}\right)\right\}+\left\{\left(v_{a}-v_{b}\right)-\left(v_{a}^{\prime}-v_{b}^{\prime}\right)\right\}+\cdots \\
+\left\{\left(v_{f}-v_{0}\right)-\left(v_{f}^{\prime}-v_{0}^{\prime}\right)\right\}=v_{i}-v_{i}^{\prime} .
\end{aligned}
$$

Because each expression in braces vanishes, $v_{i}=v_{i}^{\prime}$, and the proof is completed.

3. Maxwell's mesh equations. In actual engineering applications the junction equations are seldom employed but rather the alternative method of the mesh equations is used. A direct proof of the equivalence of the two methods does not seem to be available in the literature (we shall not consider this question either).

We now derive the mesh equations in the special case of an isolated network which can be diagramed in a plane without crossed wires. Let 
the regions (meshes) formed be numbered from 0 to $m$ (including the exterior region). Let the resistance function be defined as the inverse of the conductivity function $g(x)$, and let $r_{i j}(w)$ be the sum of the resistance functions of the wires common to region $i$ and region $j$. Let $w_{i}$ be a cyclic current flowing around region $i$. The sign of $w_{i}$ is determined by a clockwise convention for the interior regions and by a counterclockwise convention for the exterior regions. The potential is a single-valued function, so the net change in potential around the region $i$ vanishes. Thus

$$
0=\sum_{j=0}^{m} r_{i j}\left(w_{i}-w_{j}\right), \quad i=0,1, \cdots, m .
$$

These are the mesh equations for a planar network. After the fictitious currents $w_{i}$ are found, the actual currents are given immediately by the expressions $w_{i}-w_{j}$. Obviously, equations (4) are of the same form in the variables $w_{i}$ as equations (3) are in the variables $v_{i}$.

It can be shown that any distribution of current which satisfies the conservation of electricity could be realized by assigning suitable values to a set of $n$ of the cyclic currents $w_{i}$. Such a set is called a complete set $[1,6]$.

To write the mesh equations for a non-planar network, some complete set of cyclic currents is selected and a similar procedure is followed [3]. However, a theorem of MacLane [7] on "graphs" implies that some wire of a non-planar network must be traversed by at least three of the cyclic currents. This proves the following theorem.

THEOREM 4. The mesh equations for an isolated network can be expressed in the same form as the junction equations if and only if the network is planar.

To form mesh equations for a non-isolated network, it is simply necessary to add fixed non-cyclic currents which enter at one junction, follow some path, and leave at another junction.

4. Elastic networks. A proper elastic network is a set of $n+1$ junction points $(0,1, \cdots, n), n \geqq 1$, connected by springs such that each spring connects exactly two distinct junction points and no more than one spring directly connects the same two junction points. The junction points are the end points of the springs. The force function $f(x)$ of a spring is the force required to stretch a spring so that the distance between its end points is $x$. Let $f_{i j}(x)=f_{j i}(x)$ be the force function of the spring connecting junction points $i$ and $j$. If $\boldsymbol{r}_{\boldsymbol{i}}$ is the position vector of the $i$ th junction point, the components of force which the junction $i$ exerts on the spring $(i, j)$ are 


$$
\begin{aligned}
& w_{i j}^{x}=f_{i j}\left(\left|\boldsymbol{r}_{i}-\boldsymbol{r}_{j}\right|\right) \frac{x_{i}-x_{j}}{\left|\boldsymbol{r}_{i}-\boldsymbol{r}_{j}\right|}, \\
& w_{i j}^{y}=f_{i j}\left(\left|\boldsymbol{r}_{i}-\boldsymbol{r}_{j}\right|\right) \frac{y_{i}-y_{j}}{\left|\boldsymbol{r}_{i}-\boldsymbol{r}_{j}\right|}, \\
& w_{i j}^{z}=f_{i j}\left(\left|\boldsymbol{r}_{i}-\boldsymbol{r}_{j}\right|\right) \frac{z_{i}-z_{j}}{\left|\boldsymbol{r}_{i}-\boldsymbol{r}_{j}\right|} .
\end{aligned}
$$

It is convenient to express these relations in the single vector equation $\boldsymbol{w}_{i j}=\boldsymbol{g}_{i j}\left(\boldsymbol{r}_{i}-\boldsymbol{r}_{j}\right)$, where $\boldsymbol{g}_{i j}(\boldsymbol{r})$ is a vector function whose magnitude is $\left|f_{i j}(|\boldsymbol{r}|)\right|$ and whose direction in parallel to that of $\boldsymbol{r}$. Clearly $\boldsymbol{g}_{i j}(\boldsymbol{r})=-\boldsymbol{\xi}_{i j}(-\boldsymbol{r})$ if $\boldsymbol{r} \neq 0$, and to have this relation hold for $\boldsymbol{r}=0$ it must be assumed that $f_{i j}(0)=0$.

If $u_{i}$ is the total force applied to the $i$ th junction from outside, the equations of equilibrium are

$$
\boldsymbol{u}_{i}=\sum_{j=0}^{n} \boldsymbol{g}_{i j}\left(\boldsymbol{r}_{i}-\boldsymbol{r}_{j}\right), \quad i=0,1, \cdots, n .
$$

These are analogous to Maxwell's junction equations (2) but express the conservation of force rather than the conservation of electricity.

We shall say that a spring is quasi-linear if its force function $f(x)$ is a continuous, increasing, and unbounded function and if $f(0)=0$.

THEOREM 5. If $\nu$ of the junctions $(\nu \geqq 1)$ of a one piece network of quasi-linear springs are held fixed and given forces are applied to the other junctions then there is an equilibrium configuration, and this configuration is unique.

Proof. We may suppose that the junctions $(0,1, \cdots, \nu-1)$ are held fixed and that $r_{0}=0$. Define $G_{i j}(x)=\int_{0}^{x} f_{i j}(t) d t$ and

$$
\psi=\sum_{i=0}^{n} \sum_{j=0}^{n} G_{i j}\left(\left|\boldsymbol{r}_{i}-\boldsymbol{r}_{j}\right|\right)-2 \sum_{i=\nu}^{n} \boldsymbol{u}_{i} \cdot \boldsymbol{r}_{i}
$$

(Note that $G_{i j}(x)$ is the potential energy of the spring.) Then $\psi$ is a function of $3(n-\nu+1)$ variables. As before, on a $3(n-\nu+1)$-dimensional cube of edge $2 l$, one of the variables takes the value $\pm l$; for definiteness, suppose that it is the $x$ coordinates of the radius vector $\boldsymbol{r}_{i}$. Then, considering a chain sequence $i, a, b, \cdots, f, 0$,

$$
\left(x_{i}-x_{a}\right)+\left(x_{a}-x_{b}\right)+\cdots+\left(x_{0}-x_{f}\right)+\left(x_{f}-x_{0}\right)= \pm l .
$$

For some adjacent pair, say $(d, e)$, we have $\left|\boldsymbol{r}_{d}-\boldsymbol{r}_{\mathrm{o}}\right| \geqq\left|x_{d}-x_{e}\right| \geqq l / n$. Then, as before, it follows that $\psi$ is everywhere greater on a sufficiently large cube than it is at the center. Setting the partial deriva- 
tives of $\psi$ with respect to the coordinates equal to zero at a minimum point gives exactly equations (5) from $\nu$ to $n$. This proves the existence of a solution.

As in the proof of Theorem 3, one obtains the identity

$$
\begin{aligned}
& \sum_{i=0}^{n}\left(\boldsymbol{u}_{i}-\boldsymbol{u}_{i}^{\prime}\right) \cdot\left(\boldsymbol{r}_{i}-\boldsymbol{r}_{i}^{\prime}\right) \\
= & 2^{-1} \sum_{i=0}^{n} \sum_{j=0}^{n}\left\{\boldsymbol{g}_{i j}\left(\boldsymbol{r}_{i}-\boldsymbol{r}_{j}\right)-\boldsymbol{g}_{i j}\left(\boldsymbol{r}_{i}^{\prime}-\boldsymbol{r}_{j}^{\prime}\right)\right\} \cdot\left\{\left(\boldsymbol{r}_{i}-\boldsymbol{r}_{j}\right)-\left(\boldsymbol{r}_{i}^{\prime}-\boldsymbol{r}_{j}^{\prime}\right)\right\} .
\end{aligned}
$$

The expression on the left is clearly zero because either $\left(u_{i}-u_{i}^{\prime}\right)=0$ or $\left(\boldsymbol{r}_{i}-\boldsymbol{r}_{i}^{\prime}\right)=0$. Each term on the right is of the form $\left\{\boldsymbol{s}_{i j}(\boldsymbol{p})\right.$ $\left.-\boldsymbol{g}_{i j}\left(\boldsymbol{p}^{\prime}\right)\right\} \cdot\left\{\boldsymbol{p}-\boldsymbol{p}^{\prime}\right\}$, and $\boldsymbol{g}_{i j}(\boldsymbol{p})=c \boldsymbol{p}$ where $c$ is a positive scalar, $|p| c=f_{i j}(|p|)$. Thus,

$$
\begin{aligned}
\left\{\boldsymbol{g}_{i j}(\boldsymbol{p})-\boldsymbol{g}_{i j}\left(\boldsymbol{p}^{\prime}\right)\right\} & \cdot\left\{\boldsymbol{p}-\boldsymbol{p}^{\prime}\right\}=\left\{c \boldsymbol{p}-c^{\prime} \boldsymbol{p}^{\prime}\right\} \cdot\left\{\boldsymbol{p}-\boldsymbol{p}^{\prime}\right\} \\
& =c|\boldsymbol{p}|^{2}-\left(c+c^{\prime}\right) \boldsymbol{p} \cdot \boldsymbol{p}^{\prime}+c^{\prime}\left|\boldsymbol{p}^{\prime}\right|^{2} \\
& \geqq c|\boldsymbol{p}|^{2}-\left(c+c^{\prime}\right)|\boldsymbol{p}|\left|\boldsymbol{p}^{\prime}\right|+c^{\prime}\left|\boldsymbol{p}^{\prime}\right|^{2} \\
& =\left\{c|\boldsymbol{p}|-c^{\prime}\left|\boldsymbol{p}^{\prime}\right|\right\}\left\{|\boldsymbol{p}|-\left|\boldsymbol{p}^{\prime}\right|\right\} \\
& =\left\{f_{i j}(|\boldsymbol{p}|)-f_{i j}\left(\left|\boldsymbol{p}^{\prime}\right|\right)\right\}\left\{|\boldsymbol{p}|-\left|\boldsymbol{p}^{\prime}\right|\right\} \geqq 0 .
\end{aligned}
$$

Thus, each term on the right side of equation (6) is non-negative, and because the sum is zero each term must vanish. It follows that relation (7) is actually an equality and that $\left\{f_{i j}(|p|)\right.$ $\left.-f_{i j}\left(\left|\boldsymbol{p}^{\prime}\right|\right)\right\}\left\{|\boldsymbol{p}|-\left|\boldsymbol{p}^{\prime}\right|\right\}=0$. Thus, $|\boldsymbol{p}|=\left|\boldsymbol{p}^{\prime}\right|$ if $f_{i j}(x) \not \equiv 0$. But the vectors $\boldsymbol{p}$ and $\boldsymbol{p}^{\prime}$ are parallel otherwise, $\boldsymbol{p} \cdot \boldsymbol{p}^{\prime}\left\langle|\boldsymbol{p}|\left|\boldsymbol{p}^{\prime}\right| ;|\boldsymbol{p}| \neq 0\right.$ and relation (7) could not be an equality; hence $p=p^{\prime}$ if $f_{i j}(x) \neq \equiv$.

The remainder of the uniqueness proof parallels the proof of Theorem 3. Note that a similar theorem is valid for an electric network of quasi-linear conductors.

The analogy between elastic and electric networks developed here suggests a method which, though somewhat abstract, is nevertheless quite practical. The idea is simply to set up the analog of Maxwell's mesh equations for elastic networks or even for more complicated problems in statics such as trusses. Without the analogy it would appear difficult to invent such a method, as the concept of using fictitious circulating forces in the meshes, for variables, is rather fantastic.

The writer has built a mechanical model of a quasi-linear electric network (a wheatstone bridge circuit) by constraining the junctions of an elastic network to move along vertical rods. Hanging weights 
on the junctions gives "applied currents." It is clear that, even with springs obeying Hooke's law, a nonlinear problem arises.

The discrete boundary value problems discussed here suggest analogous considerations for continuous media. In a later note such nonlinear Dirichlet problems will be treated.

\section{REFERENCES}

1. W. Ahrens, Math. Ann. vol. 49 (1897) p. 311.

2. C. E. Clark, Bull. Amer. Math. Soc. vol. 47 (1941) p. 769.

3. W. Feussner, Annalen der Physik vol. 9 (1902) p. 1304, vol. 15 (1904) p. 385.

4. L. Graetz, Handbuch der Electrizität und des Magnetismus, Leipsig, 1912, vol. 2, p. 55.

5. G. P. Harnwell, Principles of electricity and electromagnetism, McGraw-Hill, 1938, pp. 138, 167.

6. G. Kirchhoff, Pogendorff Annalen vol. 72 (1847) p. 497; Collected works, p. 22.

7. S. MacLane, Fund. Math. vol. 28 (1936) p. 22; Duke Math. J. vol. 3 (1937) p. 466.

8. J. C. Maxwell, A treatise on electricity and magnetism, 3d ed., pp. 403-408.

9. Western Electric Company, Varistors: their characteristics and uses, reprints from Bell Laboratories Record.

Carnegie Institute of Technology 